\title{
EchoGéo
}

49 | 2019

Les corridors de développement

\section{L'Impact des corridors de la Région du Grand Mékong}

Hiérarchies, reconfigurations urbaines et spécialisation des fonctions politico-économiques : le cas de la ville de Chiang Raï, Thaïlande

\section{Thomas Foin}

\section{(2) OpenEdition}

\section{Journals}

Édition électronique

URL : https://journals.openedition.org/echogeo/17952

DOI : $10.4000 /$ echogeo. 17952

ISSN : 1963-1197

Éditeur

Pôle de recherche pour l'organisation et la diffusion de l'information géographique (CNRS UMR 8586)

Référence électronique

Thomas Foin, «L'Impact des corridors de la Région du Grand Mékong », EchoGéo [En ligne], 49 | 2019, mis en ligne le 25 octobre 2019, consulté le 10 août 2021. URL : http://journals.openedition.org/ echogeo/17952 ; DOI : https://doi.org/10.4000/echogeo.17952

Ce document a été généré automatiquement le 10 août 2021.

EchoGéo est mis à disposition selon les termes de la licence Creative Commons Attribution - Pas d'Utilisation Commerciale - Pas de Modification 4.0 International (CC BY-NC-ND) 


\section{L'Impact des corridors de la Région du Grand Mékong}

Hiérarchies, reconfigurations urbaines et spécialisation des fonctions politico-économiques : le cas de la ville de Chiang Raï, Thaïlande

\section{Thomas Foin}

Cet article fait suite au mémoire de recherche:

Foin T., 2017. Régionalisation et reconfigurations urbaines : La ville thailandaise de Chiang Rai. Université Paris-Diderot, Géographie, Paris, 109 p.

\section{Introduction}

1 En 1994, le gouvernement thaillandais amorce un processus de déconcentration dans l'objectif de réduire les disparités socio-économiques entre la Région Métropolitaine de Bangkok (RMB) et les régions périphériques. Ce transfert de compétences à l'échelle locale s'est effectué conjointement aux grandes stratégies planifiées par le programme de la Région du Grand Mékong (RGM). La RGM ${ }^{1}$, appuyée par la Banque Asiatique de Développement (BAD), a notamment développé, au travers d'une vision territoriale et sectorielle, l'approche des corridors économiques. Ces derniers, qui peuvent prendre diverses appellations selon leur classification, sont définis par Thein Swe et Paul Chambers (2011) comme des "couloirs économiques constituant de nouveaux territoires supranationaux au sein desquels les infrastructures de transport facilitent le développement et la croissance économique des territoires, en reliant les différents centres de production et de commerce ». Si les corridors sont désignés comme des outils d'intégration régionale $d u$ fait qu'ils transcendent les frontières et qu'ils connectent les centres économiques des pays, ils ont également pour vocation, par leur capacité à capter les flux de capitaux, de marchandises et de personnes, d'encourager le développement socio-économique des territoires traversés, principalement à l'échelle des villes. Parallèlement, des travaux scientifiques en géographie, mais aussi dans d'autres disciplines, ont démontré que la mise en réseau des villes par les corridors suscitait des relations de complémentarité et/ou de concurrence qui, par la suite, 
engendraient une nouvelle hiérarchisation des villes due à une redéfinition des fonctions urbaines (Franck M. 2014).

2 Malgré une abondante littérature académique sur les corridors, peu d'études se sont intéressées à définir les dynamiques urbaines et socio-économiques induites par les corridors à l'échelle locale. De ce constat, il a semblé opportun d'analyser les interactions entre un corridor économique et une ville de taille moyenne disposant d'une situation stratégique. La ville de Chiang Rai, capitale de la province de même nom au Nord de la Thaillande, a été retenue pour mener à bien cette recherche. Cette dernière présente l'avantage d'être d'une part sur le corridor économique Nord-Sud ${ }^{2}$ à la jonction des routes R3A (Laos) et R3B (Myanmar) et à proximité du fleuve Mékong, et d'autre part d'être en périphérie de la RMB (illustration 1).

En s'inscrivant dans la continuité des recherches sur les villes asiatiques questionnant le rôle de la globalisation dans l'émergence «de nouvelles configurations intraurbaines, réticulaires et d'échelle régionale " (Franck et Sanjuan, 2015), cet article interroge le rôle structurant du corridor Nord-Sud à l'échelle de la ville de Chiang Rai, par le prisme de nouvelles dynamiques socio-économiques et urbaines. Celui-ci s'organise autour de quatre parties, après avoir défini le cadre conceptuel sur les interactions villes/corridor il sera exposé successivement le processus d'internationalisation de la ville, l'actuel reconfiguration urbaine articulée autour du corridor Nord-Sud et enfin la nouvelle hiérarchisation des villes provinciales et régionales.

\section{Illustration 1 - Les corridors économiques de la Région du Grand Mékong}

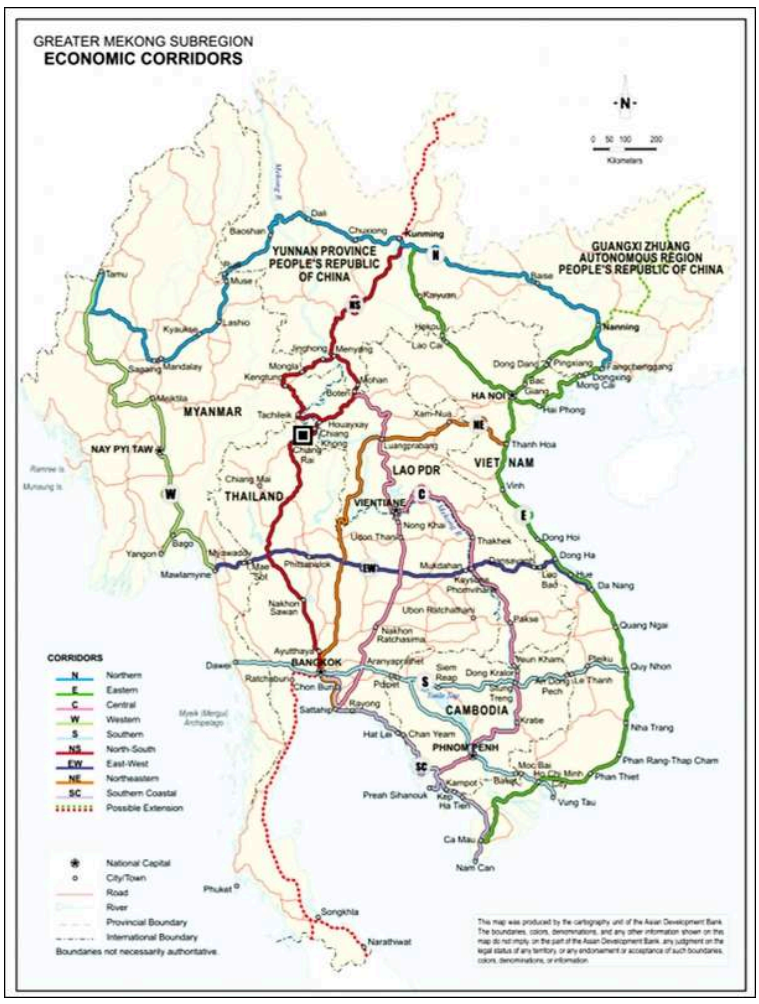

Source : ADB, 2014. 


\section{Émergence de nouvelles centralités urbaines : le modèle des villes paires}

4 Bien que les concepts « d'intégration régionale » et de "région » soient centraux dans cette recherche, les définitions qui leur sont attribuées et les différentes interprétations qui en sont faites, selon la discipline académique ou l'approche adoptée, ne seront pas débattues dans cet article. L'objectif étant de se concentrer avant tout sur les villes. Toutefois, dans un souci de clarté, il est retenu que l'intégration régionale correspond à une organisation spatiale structurant un système dont les sous-ensembles «qui le composent sont commodément reliés les uns aux autres et aux centres de pouvoirs, que toutes les parties du territoire sont correctement irriguées en services, biens, informations et moyens de travail, et que leurs produits sont efficacement drainés et redistribués » (Brunet, 2005). Enfin, le terme de région, faisant référence dans le cas précis à la RGM, est entendu comme une région transnationale assimilant ainsi les deux échelles de la notion de région, à savoir infranationale et supranationale (Taillard, 2009 et 2010).

5 Les corridors économiques de la RGM, développés au cours de deux grandes phases de planification ( $1^{\text {ère }}$ phase $1992,2^{\text {nde }}$ phase 2002), sont aujourd'hui au nombre de huit. Ce maillage permet de relier l'ensemble des états et des centres économiques de la région entre eux et d'intégrer des pays, comme la Chine, à de nouveaux marchés économiques. L'approche des corridors, soutenue par la BAD, a généré une relation d'interdépendance entre ces outils d'intégration régionale et les villes. En effet, ces dernières permettent de structurer les corridors en une série de points nodaux et participent à leur effectivité (BAD, 2014). En parallèle, les corridors favorisent l'émergence de nouveaux types de villes par l'adjonction d'activités, de fonctions gestionnaires et de flux (Franck, 2014).

6 Les villes n'ayant pas toute le même rôle ni le même poids économique et politique, plusieurs classifications ont été pensées pour les hiérarchiser. Le géographe Christian Taillard (2009 et 2010) hiérarchise ces villes en quatre catégories selon leur(s) fonction(s) et leur degré d'intégration à l'échelle nationale et régionale ${ }^{3}$. Les têtes de réseaux et les nœuds principaux combinent le plus souvent l'ensemble des fonctions économiques et politiques, à l'inverse, les nœuds secondaires et les villes relais se sont développés par une spécialisation de leurs activités économiques.

7 Pour illustrer davantage cette classification, appliquons-la au corridor Nord-Sud. Celuici s'articule entre deux têtes de réseaux, les métropoles de Kunming et de Bangkok. Ces dernières peuvent être considérées ainsi par leurs fonctions politiques et économiques à l'échelle du corridor et par leur degré d'intégration à l'échelle régionale et mondiale. En effet, Bangkok et Kunming sont chacune situées au croisement d'autres corridors ${ }^{4}$, elles jouent donc un rôle clé au sein de la RGM ainsi que dans la structuration du corridor Nord-Sud, car ce sont-elles qui influent majoritairement le développement des flux de biens et services, de capitaux et de personnes. Vient ensuite les villes définies comme nœuds principaux, par leur localisation sur le corridor, à une échelle inférieure que les têtes de réseaux, celles-ci ont assimilé des fonctions économiques et/ou politiques, qui leur procurent désormais un nouveau statut à l'échelle nationale et régionale (Franck M. 2014). C'est notamment le cas des villes de Chiang Rai et de Jinghong (Yunnan, Chine) chacune située à la jonction des routes R3A (Laos) et R3B (Myanmar) du corridor Nord-Sud. Enfin, troisième échelon significatif, les nœuds 
secondaires localisés le plus souvent le long des corridors au niveau des frontières nationales. Comme par exemple les villes frontalières de Mae Sai et Tachilek à la frontière Thaïlande-Myanmar sur la route R3B ou encore Chiang Khong et Houayxay à la frontière Thaïlande-Laos sur la route R3A. Selon les travaux d'Elsa Lainé (2013), leur modèle de développement est directement lié aux dynamiques transnationales inhérentes au passage du corridor, qui occasionnent une spécialisation de leurs fonctions (productions, échanges). Généralement, ces villes n'ont pas de fonction de gestion du corridor, toutefois leur rôle économique et leur localisation aux frontières permettent d'accroître l'efficience du corridor.

8 Le développement et la spécialisation des fonctions urbaines entraînent également des relations de complémentarités et d'interdépendances entre villes, cela étant particulièrement visible à proximité des frontières où de nouveaux schémas d'articulation se sont développés. Comme l'illustre l'illustration 2, deux modèles ont été identifiés par Manuelle Franck (2014). Tout d'abord les «villes doublons » (twin cities), comme leur nom l'indique, ces villes fonctionnent en doublon, c'est-à-dire que chacune, située de part et d'autre de la frontière, tire parti des interdépendances et complémentarités qu'offrent les deux États, la proximité est alors un facteur clé. Ensuite le modèle des "villes paires" (urban pair), ce sont également deux villes localisées dans deux États, mais à plus grande distance de la frontière que les villes doublons. Ces villes sont alors liées par des relations hiérarchiques complémentaires et/ou concurrentielles. De plus, tandis que les villes doublons sont de moins en moins tournées vers le territoire national mais davantage intégrées à l'échelle régionale, les villes paires apparaissent comme des centres de commandement directement liés aux villes frontalières.

Illustration 2 - Interactions entre villes doublons et villes paires

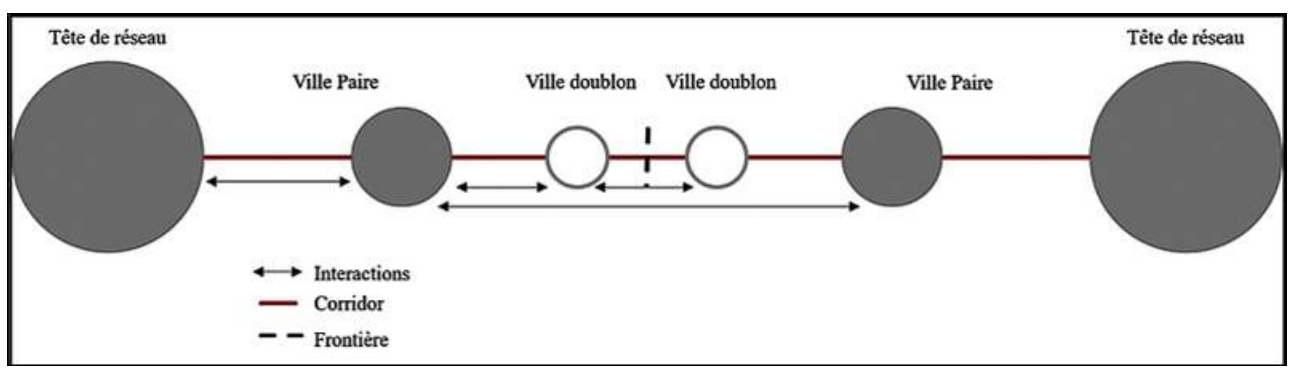

Auteur: Thomas Foin, 2017

Comme le démontre l'illustration 3, la ville de Chiang Rai, qui dispose d'une situation stratégique sur le corridor Nord-Sud, se situe à $60 \mathrm{~km}$ de la frontière avec le Myanmar et $110 \mathrm{~km}$ de celle du Laos. La province de Chiang Rai compte trois points d'entrées et de sorties, ces derniers qui correspondent aux villes frontalières de Mae Sai, Chiang Saen et Chiang Khong ont été définis par Elsa Lainé (2013) comme des villes-doublons. Enfin, en 2003, le gouvernement thaïlandais a désigné cette province pour accueillir l'une des premières Zones Économiques Spéciales (ZES). La création des ZES est une des stratégies des six états membres de la RGM pour renforcer la coopération régionale et la croissance des échanges commerciaux. De ces caractéristiques préalables, il est intéressant de comprendre comment la ville de Chiang Rai peut être spécifiée comme une ville paire. 
Illustration 3 - La province de Chiang Rai : un territoire polarisé autour des axes de transport

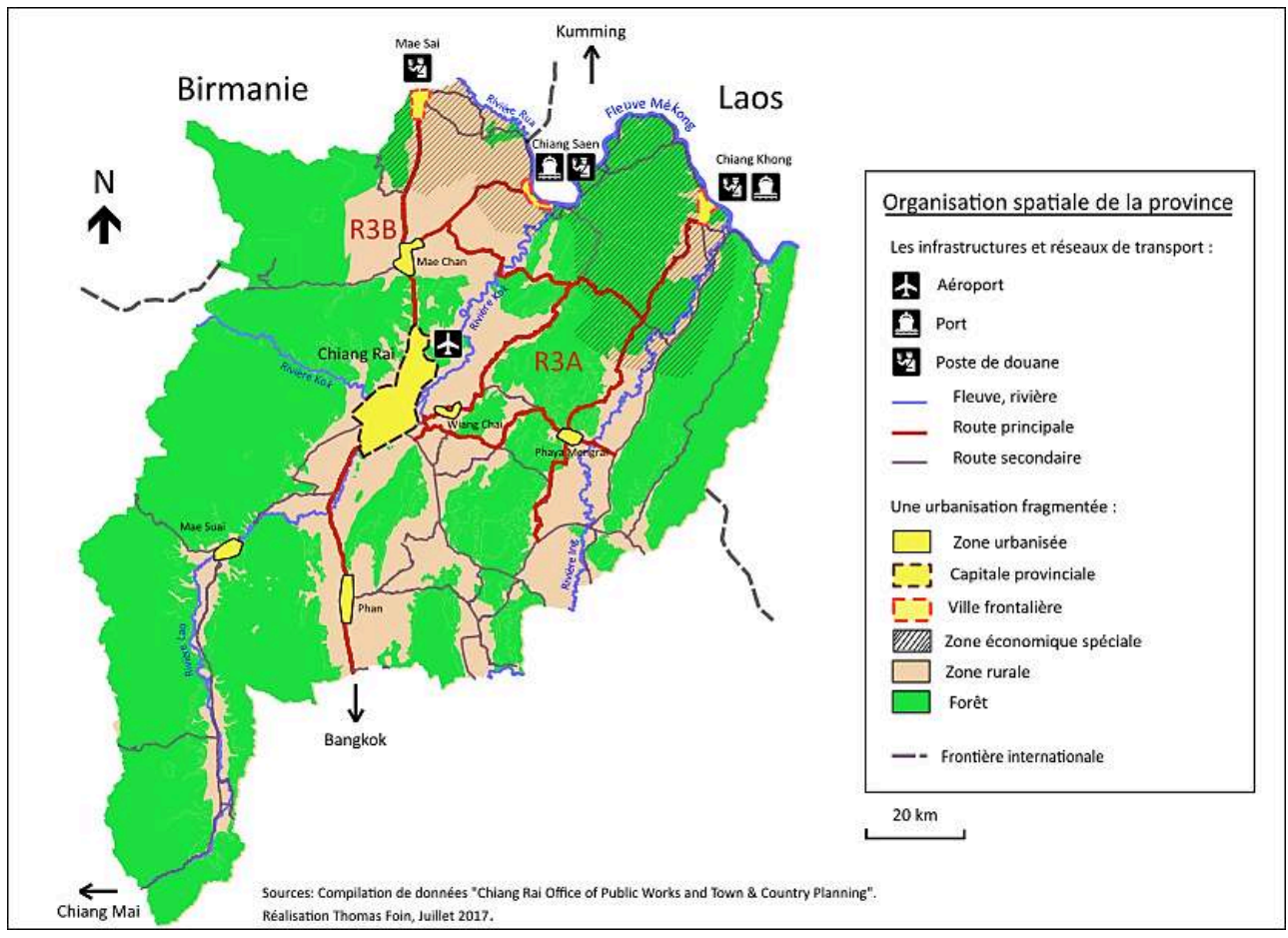

Auteur : Thomas Foin, 2017

\section{Internationalisation et polarisation des fonctions économiques}

L'approche développée par Manuelle Franck et Charles Goldblum (2007), sur l'étude des processus de métropolisation des villes de second rang, définit l'internationalisation comme l'un des critères de la métropolisation, notamment au travers des édifices (formes, lieux, fonctions) et la logique des acteurs privés (investissements tertiaires et hôteliers, spéculations foncières, etc.). À ces critères, il est possible d'ajouter d'une part la croissance des flux de biens, de personnes (migrants, touristes) et de capitaux (investissements directs étrangers, aides publiques au développement) et d'autre part la présence d'acteurs transnationaux et internationaux comme des migrants, des investisseurs ou encore des ouvriers (Lainé, 2011). Il ne s'agit pas ici d'appréhender Chiang Rai comme une métropole mais de comprendre les processus qui conduisent à l'internationaliser.

11 Le temps imparti pour cette recherche ainsi que les modalités de terrain ont conduit à spécifier les marqueurs qui rendraient le mieux compte de ce processus. Par conséquent, il a été retenu que le degré d'internationalisation serait étudié au travers des édifices et des secteurs économiques qui intègrent la ville au sein de réseaux régionaux et internationaux et qui participent à accroître son attractivité.

Tout d'abord, il est important de relever les infrastructures de transports à caractère international. La première, et sans doute la plus significative, est bien évidemment le corridor Nord-Sud qui permet de relier physiquement Chiang Rai au Laos, au Myanmar et à la Chine et par surcroît d'intensifier les investissements étrangers dans la ville. On notera également l'aéroport international de Mae Fah Luang qui assure 
quotidiennement des liaisons vers la Chine (Jinghong, Shenzhen, Changsha) et des vols intérieurs vers Bangkok, Hat Yai et Phuket.

Trois secteurs économiques, récemment développés, ont été identifiés comme marqueurs de l'internationalisation. En premier lieu le tourisme (international), en effet celui-ci permet d'insérer Chiang Rai au sein de réseaux internationaux par la mobilité et la diversité des touristes et par l'accroissement des investissements nationaux et étrangers dans ce secteur. Entre 2011 et 2016, le nombre total de visiteurs à Chiang Rai est passé de 2288218 visiteurs à 3078 976, soit une augmentation de $25,4 \%$ (Chiang Rai Office of Public Works and Town \& Country Planning). Si le corridor Nord-Sud n'est pas le seul facteur de la croissance touristique, sa capacité à renforcer les flux de marchandises et de populations a indéniablement eu des répercussions sur les mobilités touristiques nationales et internationales. Cela peut être démontré en étudiant les données statistiques des visiteurs issus des pays traversés par le corridor. Entre 2011 et 2016 le nombre de visiteurs thaïlandais a augmenté de 32,4\%. Toutefois la donnée la plus marquante se réfère aux visiteurs chinois qui, entre 2014 et 2015 (sur les mois de janviers, février et mars), ont augmenté de 51,1\%. Les visiteurs chinois arrivant majoritairement en car, le corridor a donc un impact direct sur la croissance touristique à Chiang Rai. L'augmentation du nombre de touristes s'accompagne également d'une hausse et d'une diversification des investissements dans le secteur touristique (hôtels, condotels, auberges de jeunesses, restaurants, boutiques de souvenirs, etc.) qui se concentrent principalement dans le centre historique de la ville.

14 En deuxième lieu le secteur financier, ce dernier est directement lié au corridor NordSud. Effectivement, les nouvelles fonctions de gestion et de commandement de Chiang Rai, qui seront détaillées par la suite, ont entrainé une tertiairisation de l'économie avec une croissance des investissements dans les banques et assurances. Le développement du secteur financier permet à la ville de s'inscrire dans l'économie mondiale par les flux de capitaux. Bien que les établissements banquiers soient présents dans l'ensemble de la ville, une forte concentration est située à l'Est du centre historique sur le passage du corridor.

Enfin en troisième lieu, le secteur commercial. Tout comme le tourisme ce secteur d'activité s'est fortement développé ces dernières années, principalement en raison de l'enrichissement de la ville et de ses habitants. L'édifice le plus représentatif est sans aucun doute le «Central Plaza » ouvert en 2012. Ce centre commercial appartient à une chaîne présente dans les grandes métropoles thaïlandaises comme Bangkok, Phuket ou encore Chiang Mai. Il rassemble de nombreuses enseignes internationales dans la mode, les cosmétiques, l'électronique et la restauration (exemples: Starbucks Coffee, McDonald's, Mister Donuts, etc.). Outre ce centre commercial, des supermarchés, ainsi que de nombreux concessionnaires automobiles (de marques internationales) sont apparus. La présence de ces enseignes internationales, établies le long du corridor, démontre l'intégration de la ville dans la mondialisation à travers le prisme d'une culture mondialisée. 
Illustration 4 - Corridor Nord-Sud : facteur de spécialisation et d'internationalisation des lieux économiques de la ville de Chiang Rai

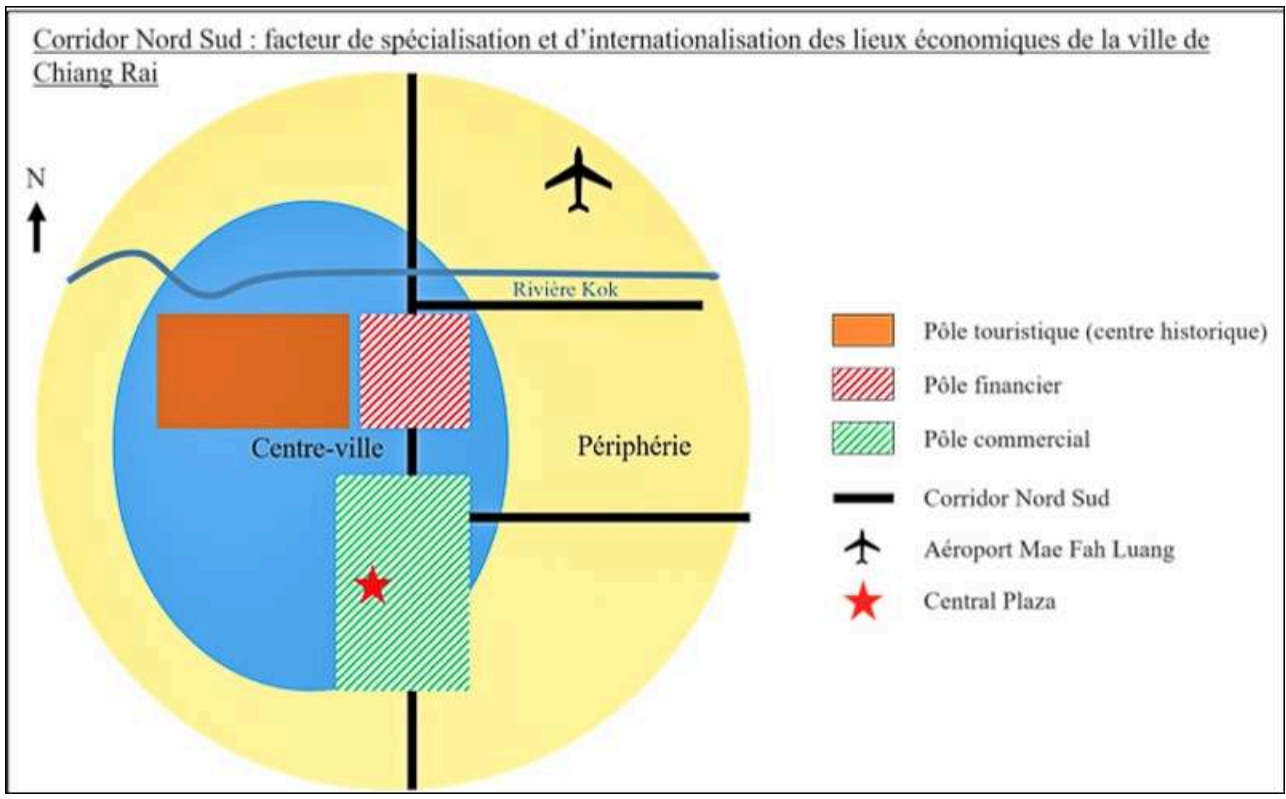

Auteur: Thomas Foin, 2017

L'illustration 4 synthétise les édifices et les lieux de l'internationalisation précédemment cités. Comme il est indiqué, le passage du corridor dans la ville tend à polariser les fonctions économiques en induisant une spécialisation des lieux. Si le centre historique combinait autrefois l'ensemble des activités économiques, dorénavant la ville s'organise autour de trois pôles: un pôle touristique, un pôle financier et un pôle commercial. En plus d'être défini comme un édifice de l'internationalisation, le corridor Nord-Sud permet, par la croissance des investissements et la captation des flux de personnes et de capitaux, la création de nouveaux pôles économiques qui d'une part étendent l'attractivité de la ville et d'autre part l'intègrent dans des réseaux internationaux. Toutefois, l'établissement du corridor et les impacts économiques sous-jacents ont inévitablement entrainé de nouvelles dynamiques urbaines et une mutation des espaces.

\section{Reconfiguration urbaine autour de nouveaux enjeux : durabilité, connectivité, intégration}

Depuis le début des années 2000, la ville de Chiang Rai a connu d'importantes transformations urbaines, dont la plus visible est l'extension des espaces bâtis le long $\mathrm{du}$ corridor. Cette urbanisation linéaire, dont les causes majeures sont le développement des investissements et la croissance démographique, s'est effectué en deux temps. Les constructions édifiées le long de la route se sont d'abord concentrées sur les bas-côtés, offrant ainsi un accès privilégié au corridor. Cet espace restreint dispose d'une fonction économique (commerces, marchés, condotels, restaurants, industries, entreprises logistiques) et publique avec la présence d'hôpitaux et d'établissements scolaires. Cependant, une fois les espaces limitrophes bâtis, l'étalement s'est élargi de part et d'autre de la route. Ce phénomène entraina une mutation des territoires. En effet, les espaces adjacents aux corridors, lorsqu'ils sont 
dépourvus de tout édifice, sont généralement des espaces agricoles. L'extension urbaine provoqua alors une mutation de ces fonctions, passant de l'agriculture à l'industrie ou à l'habitat. Si l'étalement urbain le long du corridor répond à des besoins économiques et sociaux, le manque de planification des autorités publiques a favorisé des conséquences néfastes.

18 Le potentiel économique de ces espaces a encouragé le développement de nouvelles formes d'habitat s'adressant aux classes aisées. À titre d'exemple, il est possible d'y trouver des immeubles de hauts standings ou des villas au sein de quartiers résidentiels délimités par une enceinte. Ce phénomène participe à l'embourgeoisement des périphéries et à l'attractivité de la ville, a contrario, il contribue à l'augmentation de la valeur foncière des terrains au sein de la municipalité de Chiang Rai. Dorénavant, les prix fonciers des bandes parallèles au corridor Nord-Sud peuvent égaler voire surpasser ceux du centre-ville. Par conséquent, ce processus tend à ségréguer les populations et à accroître les disparités socio-économiques en privant les habitants à faibles revenus des opportunités offertes par le corridor. De plus, comme le rappelle Suranid Ong-la, employé de la municipalité de Chiang Rai, et Tharee Kamuang, chargé d'étude (2013) : «Les mutations importantes de l'utilisation du sol, d'espaces agricoles en espaces résidentiels, impliquent le développement de nombreuses infrastructures et d'équipements. Cela a pour conséquence, sur les ressources naturelles, les forêts, l'eau, les habitats naturels et les animaux, d'entraîner une détérioration continue ». L'étalement urbain le long du corridor exerce donc une pression croissante sur les ressources naturelles, la biodiversité (déforestation, stress hydrique) et de fait sur les populations (émissions de Co2, pollution de l'eau, diminution de la surface agricole, etc.).

19 L'année 2008 marque une transition dans la pensée planificatrice de la ville de Chiang Rai. Depuis l'établissement du corridor Nord-Sud, la municipalité concevait la ville et son développement comme une porte d'entrée et de sortie vers la RGM, les aspects économiques prévalaient alors sur les aspects sociaux et environnementaux. Cependant, la dégradation des conditions de vie des habitants et les effets néfastes sur l'environnement, ont obligé la municipalité à revoir son modèle d'urbanisme. En 2008, les autorités publiques de la ville ont adopté un nouveau paradigme en matière de planification urbaine, fondé sur les concepts de développement durable, de connectivité et d'intégration (Ong-La et Kamuang, 2013). L'un des projets les plus emblématiques a été le projet Enhancing Urban Ecosystem and Biodiversity in Chiang Rai City entre 2008 et 2013. L'objectif était d'entreprendre un développement urbain établissant un cercle vertueux entre l'économie, le social et l'environnement afin d'assurer un système équitable, viable et vivable. Fondé sur cinq stratégies ${ }^{5}$, le projet a eu de nombreux impacts positifs sur la ville et sa gestion. Il a notamment encouragé la participation de la société civile à travers une approche partenariale entre le secteur public, le secteur privé, des institutions éducatives, des ONG, des scientifiques et des médias ou encore instauré le concept de développement durable dans la pensée planificatrice et les politiques de gestion de la ville.

Bien qu'ils ne soient pas nouveaux, les concepts de connectivité et d'intégration, sont aujourd'hui davantage mis en avant. Par connectivité, les pouvoirs publics n'entendent pas seulement améliorer la connexion entre les pays de la RGM via le corridor NordSud afin de dynamiser les échanges commerciaux, mais également d'optimiser la connexion à l'échelle locale, entre les zones urbaines et rurales, et à l'échelle régionale 
entre les villes (Entretien Achara Tepchai, urbaniste). Comme le souligne Jonathan Rigg et Chusak Wittayapak (2009), le développement des routes permet d'amener le marché aux habitants mais également d'amener les habitants au marché. Les routes sont donc perçues par les autorités publiques comme des outils permettant d'encourager le développement de territoires marginalisés et de limiter les disparités socioéconomiques. Quant au concept d'intégration, il est intimement lié au précédent. En effet, en développant la connectivité à diverses échelles, les autorités publiques souhaitent d'une part intégrer de nouveaux territoires à l'intérieur de l'agglomération de Chiang Rai, et d'autre part intégrer la ville au sein de la Région du Grand Mékong (RGM) afin d'accroître son rayonnement national et international.

\section{Nouvelle articulation de Chiang Rai avec les villes régionales}

21 Il a été vu précédemment que la situation géographique de la ville de Chiang Rai sur le corridor Nord-Sud avait entrainé une spécialisation des lieux au travers de son internationalisation. En parallèle, son statut de capitale provinciale et sa proximité avec le Myanmar, le Laos et la Chine ont induit une redéfinition de ses fonctions à l'échelle régionale, modifiant par conséquent les rapports hiérarchiques avec les villes de la région, principalement avec la ville de Chiang Mai et les villes frontalières de Mae Sai, Chiang Saen et Chiang Khong.

Comme l'a souligné Mr. Pattana, Président du Comité du Quadrilatère Économique ${ }^{6}$, la ville de Chiang Rai tend à devenir un centre d'affaire et de gestion. Par centre de gestion, les autorités publiques entendent faire de Chiang Rai un lieu de commandement et de coordination des stratégies économiques à mettre en place à l'échelle provinciale. Quant à centre d'affaire, la municipalité souhaite d'une part accroître le rayonnement international de la ville, notamment par la création de forums où sont conviés des acteurs publics et privés des pays de la RGM, et d'autre part concentrer les investissements au sein de la ville dans le secteur tertiaire.

L'intégration de la ville de Chiang Rai sur le corridor Nord-Sud et les nouvelles fonctions politiques et économiques qui lui sont attribuées tendent à contrebalancer les rapports hiérarchiques entre villes. En effet, la ville de Chiang Mai a toujours été la capitale économique et politique du Nord de la Thaïlande, elle est également le principal lieu touristique de cette région. Toutefois, l'essor de la ville de Chiang Rai, notamment dans le tourisme, réduit ces écarts. Comme le souligne Thein Swe et Paul Chambers (2011) : «Aujourd'hui $70 \%$ des touristes qui s'arrêtent à Chiang Mai vont ensuite à Chiang Rai ». Les données sur le tourisme confirment cette tendance, tandis que Chiang Mai est de plus en plus confrontée aux problèmes liés au tourisme de masse, la ville de Chiang Rai connaît quant à elle une forte croissance des arrivées. De plus Chiang Rai paraît aujourd'hui davantage connectée par les infrastructures de transports que sa grande sœur, aussi bien aux pays de la RGM qu'à la capitale thaïlandaise, Bangkok.

La province de Chiang Rai est considérée comme un pôle commercial du nord de la Thaïlande pour deux raisons. Premièrement, la ville de Chiang Rai apparait comme un nœud principal, disposant de fonctions économiques, politiques et logistiques, à la jonction des routes R3A et R3B et deuxièmement la localisation de la province est un atout stratégique pour le développement des échanges commerciaux sino-thaï (Sue et 
Chambers, 2011). De ce potentiel économique, le gouvernement thaïlandais a décidé d'implanter une Zone Économique Spéciale $\left(\mathrm{ZES}^{7}\right)$ au nord de la province, regroupant les districts de Mae Sai, Chiang Saen et Chiang Khong.

S'inscrivant dans l'approche des villes doublons développée par Elsa Lainé (2013), l'étude du corridor Nord-Sud et de la ZES de Chiang Rai a démontré qu'une spécialisation des activités économiques s'était effectuée entre les trois villes frontalières. Toute d'abord la ville de Mae Sai, située sur la portion R3B du corridor, elle est l'unique point d'entrée et de sortie de la province vers le Myanmar. Cette ville s'est développée en doublon avec la ville de Tachileik au Myanmar. À partir des années 2000, Mae Sai a été définie par les autorités publiques pour devenir un hub commercial où se concentreraient la production et la vente des marchandises. Ensuite la ville de Chiang Saen, celle-ci est localisée sur la route fluviale par le fleuve Mékong et ne dispose d'aucun pont terrestre pour relier le Laos. De cette situation, la ville est devenue un hub portuaire avec quatre ports dont le dernier a été inauguré en 2012. Cependant, en raison de tensions entre le Myanmar et la Chine sur le contrôle du fleuve au moment de l'étude, le port était vide (Entretien directeur adjoint des douanes, Chiang Saen). Enfin la ville de Chiang Khong qui est frontalière avec le Laos. Comme Mae Sai cette ville s'est développée en doublon avec la ville de Houayxay (Laos). Les deux villes sont reliées entre elle par un unique pont qui symbolise la route R3A du corridor Nord-Sud. C'est par cette ville que la majorité des échanges commerciaux entre la Thaïlande et la Chine transit. De fait, Chiang Khong est devenue un hub logistique.

La spécialisation de ces fonctions tend à modifier l'articulation de Chiang Rai avec les villes frontalières. Par conséquent Chiang Rai peut être définie comme une ville paire. En effet, selon le modèle théorisé par Manuelle Franck (2014), cette ville est liée par des relations hiérarchiques complémentaires aux villes frontalières. Tandis que ces dernières sont les lieux de productions et d'échanges, Chiang Rai est quant à elle un lieu de commandement et de planification des stratégies économiques. De plus, bien que la ville soit en retrait de la frontière, elle reste cependant en lien direct avec les États voisins par le biais de forums et de tables rondes.

\section{Conclusion}

Plusieurs conclusions, à différentes échelles, peuvent être extrapolées de cette étude. À l'échelle de la ville il a été démontré que le processus de régionalisation, par les corridors économiques, pouvait induire une reconfiguration urbaine des villes. La position stratégique de Chiang Rai sur le corridor Nord-Sud, à la jonction des route R3A (Laos) et R3B (Myanmar), conduit à une restructuration de la ville. Dans la perspective de tirer profit des opportunités socio-économiques apportées par le corridor, les autorités publiques en charge des politiques urbaines doivent repenser leur modèle de planification en assimilant de nouveaux concepts, tels que : développement durable, intégration et connectivité. Les enjeux principaux étant d'affirmer l'intégration régionale de Chiang Rai par l'articulation avec les villes provinciales et régionales, et d'assurer le développement socio-économique de l'agglomération par l'attraction des investissements nationaux et étrangers.

Il a été démontré que les corridors de la RGM tendent à stimuler l'essor économique des villes à travers divers processus. Dans le cas de Chiang Rai, le processus d'internationalisation de la ville et des activités économiques s'avère être le plus 
prégnant. Ce processus qui attribue de nouvelles fonctions politico-économiques et qui intègre la ville à l'échelle régionale et mondiale, peut être appréhendé comme l'amorce d'un processus global, à savoir de métropolisation (Franck et Goldblum, 2007). Si ce processus favorise la croissance économique des villes, il suscite également des rapports de dominations et de dépendances entre des centres et des périphéries, dont les routes apparaissent comme les outils d'intégration et d'exclusion.

À l'échelle provinciale et régionale, si l'établissement du corridor Nord-Sud induit l'avènement de nouvelles dynamiques territoriales locales, il entraine également une reconfiguration et une spécialisation des villes régionales, par l'adjonction de nouvelles fonctions. De son internationalisation et la tertiarisation de son économie, Chiang Rai est désormais définie comme un centre de gestion et de commandement des politiques économiques à l'échelle provinciale. Conjointement, cette redéfinition des fonctions urbaines a influencé des rapports hiérarchiques et de complémentarité entre Chiang Rai et les villes frontalières (Mae Sai, Chiang Saen, Chiang Khong), désignées comme étant des lieux de production et d'échanges (Lainé, 2013). Ainsi, dans la continuité des travaux développés par Manuelle Franck (2014), ces nouveaux rapports caractérisent Chiang Rai comme une ville paire. De plus comme il a été vu, le développement de Chiang Rai tend à contrebalancer le statut de capitale régionale de la ville de Chiang Mai.

\section{BIBLIOGRAPHIE}

Asian Development Bank (ADB), 2014. Developing Economic Corridors in South Asia. Manila.

Brunet R., 2005 ( $3^{\text {ème }}$ ed.). Les Mots de la Géographie : Dictionnaire Critique. La Documentation Française, 518 p.

Franck M., Sanjuan T., 2015. Territoires de l'Urbain en Asie. Une Nouvelle Modernité ? CNRS Editions, $404 \mathrm{p}$.

Franck M., 2014. Twin Cities and Urban Pairs, a New Level in Urban Hierarchies Structuring Transnational Corridors? A Case Study of the Pekanbaru-Dumai Urban Pair. In Fau N., Khonthapana S., Taillard C. (ed.), Transnational Dynamics in Southeast Asia, The Greater Mekong Subregion and Malacca Straits Economic Corridors. Singapore, Institute of Southeast Asian Studies ISEAS, p. 271-299.

Goldblum C., Franck M. 2007. Les Villes aux Marges de la Métropolisation en Asie du Sud-Est. L'Espace géographique, tome 36, p. 229-236.

Lainé E., 2011. Les villes frontalières thaïlandaises dans la régionalisation : planification et production de nouvelles formes urbaines. Moussons, $\mathrm{n}^{\circ} 18$, p. 51-75.

Lainé E., 2013. Le rôle des villes frontalières de la Vallée du Mékong dans la stratégie thaïlandaise de commandement de la Région du Grand Mékong. Paris, Institut National des Langues et Civilisations Orientales - INALCO, 416 p. 
Ong-La S., Kamuang T., 2013. Urban Ecosystem and Biodiversity Conservation toward Sustainable City and Climate Change Resilience. So Dreams Come True. Chiang Rai, p. 71- 81.

Rigg J., Wittayapak C., 2009. Spatial Integration and Human Transformations in the Greater Mekong Subregion. In Reshaping Economic Geography in East Asion. Washington D. C., The World Bank, p. 79-99.

Swe T., Chambers P., 2011. Cashing in Across the Golden Triangle: Thailand's Northern Border Trade with China, Laos, and Myanmar. Chiang Mai, Mekong Press, p. 142.

Taillard C., 2009. Un exemple réussi de régionalisation transnationale en Asie Orientale : les corridors de la Région du Grand Mékong. L’Espace Géographique, vol. 38, p. 1-16.

Taillard C., 2010. La Thaïlande, au Centre de la Région du Grand Mékong. Annales de Géographie, $\mathrm{n}^{\circ}$ 671-672, p. 52-68.

\section{NOTES}

1. Créé en 1992 le programme de la Région du Grand Mékong regroupe aujourd'hui cinq États d'Asie du Sud-Est continentale (Thaillande, Myanmar, Laos, Vietnam, Cambodge), la province chinoise du Yunnan et la région autonome du Guangxi (Chine).

2. Le corridor Nord-Sud relie les deux métropoles Kunming (Yunnan, Chine) et Bangkok par l'intermédiaire du Laos et du Myanmar. Planifié par la RGM et la BAD en 1992, ce corridor est l'un des plus structurants de la région.

3. La BAD classe quant à elle les villes selon leur fonction économique sur les corridors. Il en ressort quatre catégories dont : les villes commerciales, les villes frontalières, les points d'entrée et/ou de sortie (correspondant aux têtes de réseaux) et les nœuds entre axes de transport (Lord, 2009 cité par Lainé, 2013).

4. La capitale thaillandaise, à l'intersection des corridors Sud, Nord-Sud et Nord-Est et la capitale du Yunnan au croisement des corridors, Nord, Nord-Sud et Est.

5. (1) Entreprendre une politique sensible aux enjeux environnementaux et sociaux.

(2) Sensibiliser les acteurs de la planification urbaine au concept de durabilité. (3) Accorder davantage d'allocations budgétaires à la protection de l'environnement et des ressources naturelles. (4) Favoriser la participation de la société civile dans les processus décisionnels. (5) Réduire les émissions de $\mathrm{CO} 2$ et élaborer un plan d'urgence face aux catastrophes naturelles (Ong-La et Kamuang, 2013).

6. Le Comité du Quadrilatère Économique rassemble dix Chambres de Commerces du Nord de la Thailande.

7. Les ZES répondent aux objectifs soutenus par les corridors économiques en instaurant un cadre législatif qui, d'une part, doit faciliter le passage des biens et personnes aux frontières et, d'autre part, doit supporter la croissance de secteurs économiques en attirant les investissements directs étrangers. 


\section{RÉSUMÉS}

À partir des années 1990, la Région du Grand Mékong (RGM), appuyée par la Banque Asiatique de Développement (BAD), développe l'approche des corridors dans l'objectif de favoriser l'intégration régionale en Asie du Sud-Est. Ces couloirs économiques, qui relient les centres de productions et de commerces, induisent à l'échelle des villes, de nouvelles relations de complémentarité et/ou de concurrence et une redéfinition de leurs fonctions urbaines. Due à sa localisation sur le corridor Nord-Sud, la ville de Chiang Rai, située au Nord de la Thaïlande, a connu un processus de reconfiguration urbaine et une spécialisation de ses fonctions politicoéconomiques. L'étude de ces récentes dynamiques permet de définir Chiang Rai comme une ville paire.

Since the 1990s, the Greater Mekong Subregion (GMA), supported by the Asian Development Bank (ADB), has developed the corridors approach with the aim to promote regional integration in Southeast Asia. Economic corridors, which connect production and trade centers, induce at the scale of cities, new relations of complementarity and / or competition and a redefinition of their urban functions. Due to its location on the North-South Economic Corridor, Chiang Rai city, situated in northern Thailand, has undergone a process of urban reconfiguration and a specialization of its political and economic functions. The study of these recent dynamics allows to define Chiang Rai city as an urban pair.

\section{INDEX}

Mots-clés : reconfiguration urbaine, ville paire, connectivité, intégration régionale, dynamique transnationale

Keywords : urban reconfiguration, urban pair, connectivity, regional integration, transnational dynamic

Thèmes : Sur le Champ - Sur le Terrain

\section{AUTEUR}

\section{THOMAS FOIN}

Thomas Foin, foin.thomas@wanadoo.fr, est étudiant à l'Université Paris-Diderot, UFR de Géographie et a réalisé son mémoire de M2 sous la direction de Nathalie Fau. 